\title{
A INFLUÊNCIA DA AVERSÃO À PERDA NA DECISÃO DE SE SOBRECARREGAR NO TRABALHO
}

João Gabriel Modesto

Centro Universitário de Brasília

Universidade Estadual de Goiás

Tatiana Rodrigues

Centro Universitário de Brasília

Fabiana Queiroga

Centro Universitário de Brasília
Recebido em: 15/07/2019

$1^{a}$ revisão em: 08/04/2020

Aceito em: 22/05/2020

\section{RESUMO}

A presente pesquisa teve como objetivo avaliar o papel moderador do burnout na relação entre aversão à perda e tomada de decisão de se sobrecarregar no trabalho. Um total de 128 profissionais, com idades acima de 18 anos e inseridos no mercado de trabalho, responderam a uma medida de burnout e avaliaram um cenário de sobrecarga no trabalho. Verificou-se um efeito da aversão à perda na decisão de se sobrecarregar no trabalho, mas o burnout não moderou essa relação. Variáveis sociodemográficas também não influenciaram na tomada de decisão. Esse estudo evidencia o potencial heurístico da aversão à perda como mecanismo automático que pode influenciar a decisão de se sobrecarregar no trabalho. Assim, deve-se ter cautela ao expor indivíduos em processos de decisões visto que isso pode impactar negativamente tanto para o próprio indivíduo (no curto prazo), quanto para a própria organização (no médio e longo prazo).

Palavras-chave: stress ocupacional; tomada de decisão; aversão. 


\section{THE INFLUENCE OF LOSS AVERSION ON THE DECISION TO WORK OVERLOAD}

\section{ABSTRACT}

The present study aimed to evaluate the moderating role of burnout in the relationship between loss aversion and making a decision to suffer work overload. A total of 128 professionals over 18 years old inserted in the job market participated in the study, responding to a burnout measure and evaluating a work overload scenario. A loss aversion effect on the decision to suffer work overload was noted, although burnout did not moderate this relationship. Sociodemographic variables also did not influence decision making. This study highlights the heuristic potential of loss aversion as an automatic mechanism that can influence the decision to overwork at work. Thus, it is important pay attention to exposing individuals to decision-making processes, because this can impact negatively both the individual (in the short term) and the organization itself (in the medium and long term).

Keywords: occupational stress; decision making; aversion.

\section{LA INFLUENCIA DE LA AVERSIÓN A LA PÉRDIDA EN LA DECISIÓN DE SOBRECARGAR EN EL TRABAJO}

\section{RESUMEN}

El presente estudio tuvo como objetivo evaluar el papel moderador del burnout en la relación entre la aversión a la pérdida y la decisión de sufrir una sobrecarga de trabajo. Un total de 128 profesionales mayores de 18 años inscritos en el mercado laboral participaron en el estudio, respondiendo a una medida de burnout y evaluando un escenario de sobrecarga de trabajo. Se observó un efecto de aversión a la pérdida en la decisión de sufrir una sobrecarga de trabajo, aunque el burnout no moderó esta relación. Las variables sociodemográficas tampoco influyeron en la toma de decisiones. Este estudio destaca el potencial heurístico de la aversión a la pérdida como un mecanismo automático que puede influir en la decisión de trabajar en exceso en el trabajo. Por lo tanto, es importante prestar atención a exponer a los individuos a los procesos de toma de decisiones, ya que esto puede afectar negativamente tanto al individuo (a corto plazo) como a la organización misma (a mediano y largo plazo).

Palabras clave: estrés ocupacional; toma de decisiones; aversión. 


\section{INTRODUÇÃO}

Imagine duas situações que envolvem sobrecarga no trabalho. Na primeira delas você pode optar por se sobrecarregar no trabalho a fim de evitar perder o padrão de vida que possui. Na segunda situação você pode optar por se sobrecarregar para receber vencimentos maiores e incrementar seu padrão de vida. Em qual das situações você acha mais provável que as pessoas em geral optem por se sobrecarregar no trabalho? Quais vieses cognitivos estão em jogo nesta tomada de decisão? Uma pessoa que já está esgotada no trabalho está menos propensa a se sobrecarregar? Buscando analisar possíveis fatores associados a uma tomada de decisão no contexto laboral, a presente pesquisa teve como objetivo avaliar o papel moderador do burnout na relação entre aversão à perda e tomada de decisão de se sobrecarregar no trabalho.

\section{ESGOTAMENTO NO TRABALHO E SOBRECARGA LABORAL}

A partir do trabalho de um psicólogo clínico (Herbert Freudenberge) e de uma psicóloga social que estuda emoções no trabalho (Christina Maslach) aparecem na década de 1970 as primeiras publicações sobre burnout (Aronsson et al., 2017). Em 2015, no editorial da revista Research Burnout, Maslach e seu companheiro de pesquisa Michael Leiter identificaram que havia, naquele período, mais de 1000 artigos versando sobre vários aspectos do burnout, publicados todos anos. O volume de pesquisas e meta-análises internacionais sobre o assunto (p. ex. Ahola, Toppinen-Tanner, Seppänen, 2017; Alarcon, 2011; Dreison et al., 2018) legitimam o interesse dos pesquisadores pela temática. Contudo, na literatura nacional, a produção do conhecimento sobre o fenômeno ainda é considerada instável (ver Dalcin \& Carlotto, 2018 para uma agenda de pesquisa sobre burnout em professores). De uma forma geral, há grande consenso sobre as características principais da síndrome de burnout (Hyeda \& Handar, 2011; Maslach \& Jackson, 1981). Um dos pontos comuns abordados por tais pesquisadores é que a síndrome está relacionada ao estresse e esgotamento emocional. Além disso, é pacificado que, diferentemente do estresse, o burnout sempre tem caráter negativo para o indivíduo e está relacionado ao ambiente laboral. Portanto, o burnout é um processo que causa prejuízos ao trabalho no exercício das suas atividades laborais e traz diversas implicações para o indivíduo, a exemplo da depressão (De Francisco, Arce, Vílchez, \& Vales, 2016), bem como impactos negativos para a organização, como a redução do desempenho e da satisfação no trabalho (Fogarty, Singh, Rhoads, \& Moore, 2000).

De maneira resumida, é possível descrever que o burnout tem seu surgimento a partir do estresse ocupacional e do fracasso de se estabelecer estratégias de coping eficientes (Aronsson et al., 2017). Assim, a discrepância entre as demandas ambientais e os recursos individuais para enfrentá-las iniciam a percepção de estresse ocupacional. Contudo, quando a busca de recursos para diminuir essa discrepância mobiliza o trabalhador a desenvolver-se profissional e pessoalmente observa-se que a situação estressora atuou positivamente. Por outro lado, 
quando os recursos empregados são falhos, e a situação estressora perdura, acarreta no indivíduo o processo de burnout (Dreison et al., 2018; Sonnentag \& Fritz, 2007).

Em um trabalho sobre as implicações metodológicas da tradução do termo burnout, Squires et al. (2014) identificam que, em termos de definição do construto, a mais conhecida e utilizada sobre burnout é a de Maslach e Jackson (1981) que apontam três fatores principais: a) exaustão emocional caracterizada por uma falta ou carência de energia, entusiasmo e um sentimento de esgotamento de recursos; b) despersonalização que tem por definição tratar organizações, colegas e clientes como objetos; c) realização pessoal que é a tendência do trabalhador se auto avaliar de forma negativa o que faz a pessoa se sentir insatisfeita com seu desenvolvimento profissional. Ainda que essa definição tenha sido atualizada (Maslach, Schaufeli, \& Leiter, 2001), o burnout continua sendo definido como um fenômeno psicossocial de prolongada exaustão tipicamente associado ao contexto de trabalho.

Não obstante, várias são as críticas feitas a esse modelo e uma delas é a de que a dimensão de exaustão ignora aspectos relacionados ao físico e ao cognitivo vivenciado pelo trabalhador. Assim, no final da década de 1990, modelos como o de Demerouli, Backer, Nachreineir e Shaufeli (2001) procuram superar essa lacuna e propõem, para a mensuração do burnout, que exaustão fique entendida como uma consequência da intensa pressão, afetiva e física, ou seja, uma consequência de longo prazo às exigências consideradas como desfavoráveis de trabalho. Além disso, adiciona-se nos modelos de definição do burnout o desligamento do trabalho, isto é, o distanciamento do objeto e do conteúdo do trabalho, particularmente com a vontade de continuar na mesma profissão ou organização (Shuster \& Dias, 2018). Nesse sentido, o presente trabalho busca incorporar tais atualizações conceituais com vistas a ampliar a interpretação dos resultados.

Pesquisas anteriores têm investigado antecedentes e consequentes do burnout em diferentes níveis de análise (Ahola, Toppinen-Tanner, Seppänen, 2017; Carrasco, Martínez-Tur, Peiró, García-Buades, \& Moliner, 2011; Dreison et al., 2018). O mais comum, conforme já foi ressaltado, é a análise do burnout como sendo uma consequência do estresse ocupacional (Maslach, Schaufeli, \& Leiter, 2001; Squires et al., 2014) e que esse construto seja investigado entre professores e profissionais da saúde (Dreison et al., 2018; Schaufeli, Leiter \& Maslach, 2009). Todavia, diante das suas características, esse é um construto que pode aparecer em qualquer categoria profissional.

Para fins da presente pesquisa, ressalta-se a importância de analisar o excesso de demandas de trabalho (Aronsson, 2017), na medida em que o burnout pode ser entendido como uma reação a uma sobrecarga de trabalho (Carlotto, Dias, Batista, \& Diehl, 2015). Mais especificamente, o interesse, na presente pesquisa, recai sobre uma tomada de decisão do indivíduo em aceitar novas demandas e se sobrecarregar no trabalho. Embora em muitas situações o indivíduo não tenha 
autonomia para tomar essa decisão, nos casos em que é dada ao indivíduo a possibilidade de decidir, quais fatores podem influenciar nesse processo decisório? Existem vieses cognitivos, a exemplo da aversão à perda, que podem interferir em uma decisão mais deliberada e racional? O nível atual de burnout do indivíduo interfere nesse processo decisório?

\section{AVERSÃO À PERDA}

Um amplo conjunto de pesquisas baseado na Teoria dos Prospectos tem evidenciado que o processo de tomada de decisão possui uma série de vieses, a exemplo da aversão à perda (Barberis, 2013 como uma revisão). Este viés indica que as pessoas, em geral, são mais sensíveis às perdas do que aos ganhos (Tversky \& Kahneman, 1992) quando decidem algo para si próprio (ver Füllbrunn \& Luhan, 2017 para análise da aversão à perda na decisão para outras pessoas), sendo este um processo que ocorre de maneira automática e não consciente (Kahneman, 2012; Modesto, 2018).

O mecanismo de aversão à perda teria origem na evolução da espécie, tendo em vista que organismos que percebiam perdas como mais urgentes do que oportunidades de ganhos teriam uma maior chance de sobreviver e se reproduzir (Kahneman, 2012). Mais recentemente, tem-se buscado identificar as bases neurais do viés, em que se tem identificado, por exemplo, que a norepinefrina, (Sokol-Hessner \& Rutledge, 2019) e que respostas neurais em regiões do cérebro estriado, somatossensorial e límbico estariam relacionados com o fenômeno (Canessa et al., 2017).

Para além da compreensão das bases evolucionista e neurais, têm sido analisadas as diferenças individuais na ocorrência do viés. Nesse sentido, há evidências que maiores índices de consciensciosidade (Boyce, Wood, \& Ferguson, 2016), bem como experiências individuais com ganhos e perdas podem atuar como fator explicativo da origem do viés (Walasek \& Stewart, 2015). Sobre este último aspecto, verificou-se que, em função destas experiências individuais, a pessoa pode desenvolver uma maior aversão à perda, neutralidade à perda ou até mesmo um efeito inverso à aversão à perda.

Outro eixo de investigação se refere à compreensão do papel exercido pela cultura na compreensão do viés. Tendo como base o Modelo Cultural de Hofstede (Hofstede, 1980), verificou-se, em um estudo desenvolvido em 53 países, que maiores índices de individualismo, distância do poder e masculinidade estão associados a maiores índices de aversão à perda (Wang, Rieger, \& Hens, 2017). Apesar das diferenças individuais e culturais mencionadas, o fenômeno é robusto interferindo em um processo decisório em diferentes contextos (Heilman, Green, Reddy, Moss, \& Kaplan, 2017; Mannor, Wowak, Bartkus, \& Gomez-Mejia, 2016; Schindler \& Pfattheicher, 2017; Valle \& Ruz, 2015).

Especificamente sobre o contexto das organizações e trabalho, são evidenciados os impactos do viés em diferentes situações. Por exemplo, considerando que a 
escolha final de candidatos em um processo seletivo pode não ser integralmente racional e consciente (Highhouse, 1997), foi identificado que a aversão à perda pode influenciar nessa escolha (Highhouse \& Johnson, 1996). Os autores criaram dois experimentos que envolviam tomada de decisão sobre finalistas em um processo seletivo, em que foi verificado que o candidato que apresentava uma queda em seu desempenho (de uma etapa para outra) foi avaliado mais negativamente do que o candidato com um desempenho regular (porém constante nas diferentes etapas) o que, de acordo com os autores, tem uma relação com a aversão à perda.

Em um estudo com altos executivos de diferentes organizações (Mannor et al., 2016), buscou-se analisar a influência dos níveis de ansiedade dos executivos em tomadas de decisão de risco. De acordo com os autores, o esperado era que executivos mais ansiosos priorizassem estratégias de menor risco em suas decisões. Apesar dessa hipótese geral, verificou-se que quando uma situação era avaliada como de potencial perda, em função do viés, tanto executivos com alta como aqueles com baixa ansiedade optaram por decisões arriscadas, mas que poderiam prevenir potenciais perdas. Esses dados indicam o impacto da aversão à perda no processo decisório mesmo de profissionais do alto escalão de organizações.

Outro aspecto que tem se mostrado relevante para o contexto das organizações envolve uma tomada de decisão ética. Nesse âmbito, verifica-se que, em situações de potenciais perdas financeiras, as pessoas estariam mais propensas a agir de maneira desonesta se comparado a uma situação que começariam a receber os benefícios (Grolleau, Kocher, \& Sutan, 2016; Modesto, 2018; Schindler \& Pfattheicher, 2017). Nesse sentido, a aversão à perda favoreceu com que as pessoas optassem por decisões desonestas.

Conforme descrito, estudos anteriores apresentaram evidências da importância que o fenômeno pode ter em processos decisórios no contexto das organizações e trabalho. No entanto, não encontramos na literatura estudos que tenham buscado analisar esse processo decisório no que se refere à saúde do trabalhador e, mais especificamente, na decisão de se sobrecarregar no trabalho. Buscando sanar essa lacuna da literatura, conforme mencionado, a presente pesquisa teve como objetivo avaliar o papel moderador do burnout na relação entre aversão à perda e tomada de decisão de se sobrecarregar no trabalho. Formulou-se como hipótese $(\mathrm{H} 1)$ que a aversão à perda irá incrementar a decisão do indivíduo de se sobrecarregar no trabalho, sendo este efeito moderado pelo grau de esgotamento atual (burnout) do indivíduo $(\mathrm{H} 2)$.

\section{MÉTODO}

\section{PARTICIPANTES}


Participaram do presente estudo 128 brasileiros, sendo a maioria do sexo feminino (62,20\%). As idades variaram entre 19 e 74 anos $(M=37,95 ; D P=$ 10,39). A escolaridade oscilou de ensino fundamental incompleto até o nível de doutorado, sendo a maior parcela dos participantes com ensino superior completo (34,38\%). Em relação à inserção profissional, 53,97\% declararam atuar no serviço público e 43,65\% na iniciativa privada. O restante da amostra (2,38\%) foi composto por profissionais autônomos. Foi uma amostra de conveniência (não-probabilística) em que os únicos critérios para participar da pesquisa foram ter mais de 18 anos e estar exercendo alguma atividade profissional no período em que ocorreu a pesquisa.

\section{INSTRUMENTOS}

Foram utilizados dois cenários, um para cada condição (ganhos vs perdas). Os cenários apresentavam, inicialmente, uma situação hipotética comum a ambas as condições:

Suponha que você é funcionário de uma conceituada empresa no país. Em função de remanejamento de pessoal, você recebe uma proposta de mudança de função com um salário mais alto. Nesta nova função, a sua carga de trabalho será bastante elevada e te obrigará a estar à disposição da organização, praticamente inviabilizando uma interação mais aprofundada com família e amigos por tempo indeterminado. Você tem autonomia para ficar na função anterior ou mudar de função.

Após a apresentação da situação genérica, havia a manipulação da aversão à perda:

Condição de ganho: Considere que a sua situação socioeconômica atual é estável, e que seu salário atual é suficiente para você manter o seu padrão de vida. No entanto, os vencimentos para a nova função te permitiriam ganhar um novo padrão de vida, tornando possível, por exemplo, que você troque de carro, além de outros ganhos.

Condição de perda: Considere que a sua situação socioeconômica atual é instável, e que seu salário atual não é suficiente para você manter o seu padrão de vida. No entanto, os vencimentos para a nova função te permitiriam evitar perder seu padrão de vida atual, tornando possível, por exemplo, que você não atrase o financiamento do carro, além de evitar outras perdas.

Cada participante foi apresentado a um dos cenários (condição de ganho ou condição de perda) e, em escala dicotômica, teve que decidir se aceitaria ou não se sobrecarregar no contexto laboral. Os cenários foram desenvolvidos pela equipe de pesquisadores e submetidos a pré-teste, tendo sido identificada a adequação para a realização do presente estudo. No pré-teste, foi analisada a adequação semântica e capacidade do cenário representar o fenômeno esperado. 
Os participantes responderam também à Escala de Caracterização de Burnout (ECB) cuja proposta é fazer a adaptação do Inventário de Burnout de Maslach (MBI) para o contexto brasileiro (Tamayo \& Tróccoli, 2009) e que é composto originalmente por 35 itens. No entanto, a escala utilizada, na presente pesquisa, foi composta por 24 itens. Os itens referentes ao Fator II, relacionados à desumanização no contexto de saúde, foram retirados, uma vez que não fez parte do escopo desse trabalho a restrição da investigação sobre uma determinada categoria profissional. A escala apresentou índice satisfatório de consistência interna $(\alpha=0,90)$. Para análise dos escores desse instrumento, 0 burnout foi considerado como uma variável contínua (conforme orientações de Benevides-Silva, 2002 que também mensura esse construto a partir do MBI).

\section{PROCEDIMENTO DE COLETA DE DADOS}

Inicialmente o projeto foi submetido e aprovado pelo Comitê de Ética e Pesquisa, sendo aprovado sob o número do parecer 2.647.521. Após a aprovação, iniciouse a coleta. Pessoas, em diferentes locais públicos da cidade de Brasília foram convidadas a participar de uma pesquisa sobre tomada de decisão sobre questões relacionadas ao contexto de trabalho. Uma vez checados os critérios para participação, foram informados aspectos éticos da pesquisa, a exemplo da garantia de sigilo e anonimato. Tendo o aceite do indivíduo por meio do Termo de Consentimento Livre e Esclarecido, o participante era alocado, de maneira aleatória, em uma das condições do estudo (ganhos vs perdas). Independente da condição, o participante respondeu à ECB, lia o cenário e tomava a decisão sobre se sobrecarregar no trabalho. Após indicar, em escala dicotômica, a decisão se aceitaria ou não se sobrecarregar, os participantes informaram dados sociodemográficos.

\section{PROCEDIMENTO DE ANÁLISE DE DADOS}

Considerando a natureza dicotômica da variável critério (decisão de se sobrecarregar no trabalho: $\operatorname{sim} X$ não), foram conduzidas regressões logísticas (quando a variável preditora foi contínua) e o teste qui-quadrado (para relacionamento com variáveis categóricas) com Intervalo de Confiança (IC). Para todos os testes estatísticos, utilizou-se como valor de referência o nível de significância de $5 \%$. Especificamente para o teste de moderação, foram seguidas recomendações da literatura (Aiken \& Stephen, 1991) para o seu teste por meio da regressão linear.

\section{RESULTADOS}

Em primeiro lugar, testou-se por meio de um Qui-quadrado, a hipótese da influência da aversão à perda na decisão de sobrecarregar no trabalho. Conforme visualizado na Tabela 1, corroborou-se a hipótese de que, a fim de evitar perder um padrão de vida atual, as pessoas optaram por se sobrecarregar mais, se 
comparado a uma situação em que incrementariam o padrão de vida, $\chi^{2}(1)=$ 7,82, $p=0,005$ (OR=0,36; IC-95\%: 0,17-0,74).

\section{Tabela 1.}

Frequências de decisão pela sobrecarga de trabalho comparando ganhos e perdas.

\begin{tabular}{c|c|c|c} 
Tipo & Sobrecarga & Não sobrecarga & Total \\
\hline Ganho & $20(32,26 \%)$ & $42(67,74 \%)$ & 62 \\
Perda & $36(57,14 \%)$ & $27(42,86 \%)$ & 63 \\
\hline
\end{tabular}

Após a identificação do efeito da aversão à perda na sobrecarga de trabalho, testou-se o efeito direto do burnout na decisão de se sobrecarregar. Por meio de uma regressão logística, não foram encontrados efeitos diretos do burnout na decisão de se sobrecarregar $(\beta=0,04, p=0,900)$. Para testar 0 efeito de moderação, a variável contínua foi centralizada (Aiken \& Stephen, 1991), sendo então criado um termo de interação entre a variável categórica (ganhos vs perdas) e o índice de burnout. Não foi encontrado um efeito significativo da variável moderadora na decisão de sobrecarregar $(\beta=0,01, p=0,981)$.

Em seguida, testamos também os efeitos de variáveis demográficas na decisão de se sobrecarregar no trabalho. Em relação à influência do sexo, não foram encontradas diferenças significativas, $\chi^{2}(1)=1,45, p=0,228$. Sobre a influência da escolaridade, foi encontrado um efeito marginalmente significativo $\beta=0,33, p=$ 0,060, indicando que quanto maior a escolaridade maior é a intenção de se sobrecarregar no trabalho.

Optou-se também em comparar a decisão de se sobrecarregar no trabalho no âmbito do serviço público em relação ao da iniciativa privada. Nesse quesito, optou-se por não analisar os dados dos participantes que se declararam autônomos em função de seu baixo percentual na presente amostra.

\section{Tabela 2.}

Frequências de decisão pela sobrecarga de trabalho no âmbito do serviço público comparada com o da iniciativa privada.

\begin{tabular}{ccc|c} 
Trabalho & Sobrecarga & Não sobrecarga & Total \\
\hline Iniciativa Privada & $28(53,85 \%)$ & $24(46,15 \%)$ & 52 \\
Serviço Público & $26(38,24 \%)$ & $42(61,76 \%)$ & 68 \\
\hline
\end{tabular}

Não foram encontradas diferenças significativas nesta comparação, $\chi^{2}(1)=2,90, p$ $=0,089$, conforme pode ser visualizado na Tabela 2 . Esses resultados serão discutidos considerando a literatura contemplada. 


\section{DISCUSSÃO}

A presente pesquisa teve como objetivo principal avaliar o papel moderador do burnout na relação entre aversão à perda e tomada de decisão de se sobrecarregar no trabalho. Uma das hipóteses formuladas $(\mathrm{H} 1)$ foi a de que a fim de evitar perder um padrão de vida que já possui, um indivíduo estaria mais propenso a se sobrecarregar no trabalho se comparado a uma situação de incremento de benefícios\ padrão de vida. A segunda hipótese $(\mathrm{H} 2)$ postulava que o efeito da aversão à perda seria moderado pelo grau de esgotamento no trabalho (burnout) do indivíduo.

A Hipótese 1 foi corroborada. A fim de evitar perder os benefícios que já possui, as pessoas indicaram uma maior intenção de se sobrecarregar no trabalho se comparado a uma situação de incremento dos benefícios. Alguns estudos têm evidenciado que as pessoas são, em geral, em torno de duas vezes mais sensíveis às perdas do que aos ganhos (Barberis, 2013; Tversky \& Kahneman, 1992), o que ajuda a compreender os resultados da presente pesquisa. $O$ incômodo gerado pela possibilidade de perder o padrão de vida que possui favoreceu com que os indivíduos optassem por se sobrecarregar no trabalho em maior grau se comparado àqueles que poderiam se sobrecarregar a fim de incrementar seus benefícios financeiros. Esses achados indicam o potencial heurístico da aversão à perda para a compreensão do processo de tomada de decisão no momento de se sobrecarregar no trabalho, contribuindo com os achados sobre viés em processos que envolvem o contexto organizacional (Highhouse, 1997; Highhouse \& Johnson, 1996; Mannor et al., 2016). Ressalta-se que, tendo em vista que a aversão à perda pode ser considerada um processo automático (Kahneman, 2012), esses dados evidenciam que a decisão de se sobrecarregar pode não ser inteiramente consciente e deliberada, possuindo vieses que interferem nessa decisão.

Sobre a segunda hipótese $(\mathrm{H} 2)$, diferente do esperado, o índice de esgotamento do indivíduo não teve um efeito direto na decisão de se sobrecarregar, nem moderou o efeito encontrado em $\mathrm{H} 1$. Esse dado é alarmante ao indicar que uma pessoa, mesmo com altos índices de esgotamento associado ao trabalho (Maslach \& Leiter, 2017), pode optar por se sobrecarregar. Organizações podem levar esse dado em consideração no momento de oportunizar certas decisões aos seus colaboradores que podem ter um impacto negativo em seus bemestares.

Sobre a influência de variáveis sociodemográficas, não foi encontrado efeito. Ainda que a variável escolaridade tenha apresentado um efeito marginal, variáveis como gênero ou inserção profissional (iniciativa privada ou serviço público) não apresentaram efeito significativo na decisão de se sobrecarregar no trabalho, o que chama mais atenção para a robustez da aversão à perda como variável preditora do fenômeno. Outras contribuições e algumas limitações da pesquisa são discutidas a seguir. 


\section{CONSIDERAÇÕES FINAIS}

Em uma perspectiva teórica, evidencia-se, com a presenta pesquisa, o potencial heurístico da aversão à perda como mecanismo automático que pode influenciar a decisão de se sobrecarregar no trabalho. Esse achado se soma a um conjunto de pesquisas que demonstram a importância de se levar em consideração o mecanismo de aversão à perda em processos decisórios (Grolleau et al., 2016; Modesto, 2018; Schindler \& Pfattheicher, 2017). Em uma perspectiva mais aplicada, organizações podem, com base nesses achados, ter cautela ao expor indivíduos em processos de decisões que podem impactar negativamente tanto para o próprio indivíduo (no curto prazo), quanto para a própria organização (no médio e longo prazo).

Apesar das evidências, a presente pesquisa possui limitações. Tais achados podem ter variações entre categorias profissionais específicas, o que não foi considerado nesse estudo, já que foram avaliados quaisquer trabalhadores em geral. É possível que diferentes cargos\funções divirjam na intensidade do viés de aversão à perda. Outra questão a ser tratada, ainda com referência aos participantes, é a composição homogênea quanto às características culturais, visto que participaram apenas respondentes de uma região do contexto brasileiro. Sugere-se que novas pesquisas investiguem também outros contextos culturais, uma vez que a psicologia de maneira geral, e a psicologia social em específico, devem levar em conta aspectos contextuais na análise dos fenômenos psicológicos (Pettigrew, 2018).

Assim, sugere-se que novas pesquisas possam focar em profissões específicas, elaborando cenários mais típicos para a categoria profissional analisada e levar em conta outras características do perfil dos participantes não consideradas na presente pesquisa. Além disso, considera-se relevante analisar o impacto de aspectos contextuais considerando, para tanto, a ampliação das características culturais dos participantes.

\section{REFERÊNCIAS}

Aiken, L. S., \& Stephen, W. G. (1991). Multiple regression: Testing and Interpreting Interactions. Thousand Oaks: Sage Publications.

Alarcon, G. M. (2011). A meta-analysis of burnout with job demands, resources, and attitudes. Journal of Vocational Behavior, 79(2), 549-562. doi:10.1016/j.jvb.2011.03.007

Aloha, K., Toppinen-Tanner, S. \& Seppänen, J. (2017). Interventions to alleviate burnout symptoms and to support return to work among employees with burnout: Systematic review and meta-analysis. Burnout Research, 4, 1-11. doi:10.1016/j.burn.2017.02.001

Aronsson, G., Theorell, T., Grape, T., Hammarström, G., Hogstedt, C., ... Hall, C. (2017). A systematic review including meta-analysis of work environment and burnout symptoms. BMC Public Health, 17, 1-13. doi:10.1186/s12889-017-4153-7

Barberis, N. C. (2013). Thirty years of Prospect Theory in economics: A review and assessment. Journal of Economic Perspectives, 27(1), 173-196. doi:10.1257/jep.27.1.173

Benevides-Silva, A. M. T. (2002). Burnout: O proceso de adoecer pelo trabalho. In A. M. T. BenevidesSilva (Ed.), Burnout: quando o trabalho ameaça o bem-estar do trabalhador (pp. 21-91). São Paulo, SP: Casa do Psicólogo. 
Boyce, C. J., Wood, A. M., \& Ferguson, E. (2016). Individual differences in loss aversion: Conscientiousness predicts how life satisfaction responds to losses versus gains in income. Personality and Social Psychology Bulletin, 42(4), 471-484. doi:10.1177/0146167216634060

Canessa, N., Crespi, C., Baud-Bovy, G., Dodich, A., Falini, A., Antonellis, G., \& Cappa, S. F. (2017) Neural markers of loss aversion in resting-state brain activity. NeuroImage, 146, 257-265. doi:10.1016/j.neuroimage.2016.11.050

Carlotto, M. S., Dias, S. R. da S., Batista, J. B. V., \& Diehl, L. (2015). O papel mediador da autoeficácia na relação entre a sobrecarga de trabalho e as dimensões de Burnout em professores. Psico-USF, 20(1), 13-23. doi:10.1590/1413-82712015200102

Carrasco, H., Martínez-Tur, V., Peiró, J. M., García-Buades, E., \& Moliner, C. (2011). Service climate and display of employees' positive emotions: The mediating role of burnout and engagement in services. Psychologica, 55, 229-253.

Dalcin, L., \& Carlotto, M. S. (2018). Síndrome de burnout em professores no Brasil: Considerações para uma agenda de pesquisa. Psicologia em Revista, 23(2), 745-770. doi:10.5752/P.16789563.2017v23n2p745-770

De Francisco, C., Arce, C., Vílchez, M. del P., \& Vales, Á. (2016). Antecedents and consequences of burnout in athletes: Perceived stress and depression. International Journal of Clinical and Health Psychology, 16(3), 239-246. doi:10.1016/j.jjchp.2016.04.001

Demerouli, E., Backer, A. B., Nachreineir, F., \& Shaufeli, W. B. (2001). The job demands-resouces model of burnout. Journal of Applied Psychology, 86(3) 499-512. doi:10.1037/00219010.86.3.499

Dreison, K. C., Luther, L., Bonfils, K. A., Sliter, M. T., McGrew, J. H., \& Salyers, M. P. (2018). Job burnout in mental health providers: A meta-analysis of 35 years of intervention research. Journal of Occupational Health Psychology, 23(1), 18-30. doi:10.1037/ocp0000047

Fogarty, T. J., Singh, J., Rhoads, G. K., \& Moore, R. K. (2000). Antecedents and consequences of burnout in accounting: Beyond the role stress model. Behavioral Research in Accounting, 13, 31-67.

Füllbrunn, S. C., \& Luhan, W. J. (2017). Decision making for others: The case of loss aversion. Economics Letters, 161, 154-156. doi:10.1016/j.econlet.2017.09.037

Grolleau, G., Kocher, M. G., \& Sutan, A. (2016). Cheating and loss aversion: Do people cheat more to avoid a loss? Management Science, 62(12), 3428-3438. doi:10.1287/mnsc.2015.2313

Heilman, R. L., Green, E. P., Reddy, K. S., Moss, A., \& Kaplan, B. (2017). Potential impact of risk and loss aversion on the process of accepting kidneys for transplantation. Transplantation, 101(7), 1514-1517. doi:10.1097/TP.0000000000001715

Highhouse, S. (1997). Understanding and improving job-finalist choice: The relevance of behavioral decision research. Human Resource Management Review, 74), 449-470. doi:10.1016/S1053-4822(97)90029-2

Highhouse, S., \& Johnson, M. A. (1996). Gain/Loss asymmetry and riskless choice: Loss Aversion in choices among job finalists. Organizational Behavior and Human Decision Processes, 68(3), 225-233. doi:10.1006/obhd.1996.0101

Hofstede, G. (1980). Culture's consequences comparing values, behaviors, institutes and organizations across nations. Beverly Hills: Sage.

Hyeda, A., \& Handar, Z. (2011). Avaliação da produtividade na síndrome de Burnout. Revista Brasileira de Medicina do Trabalho, 9(2), 78-84.

Kahneman, D. (2012). Rápido e devagar: duas formas de pensar. Rio de Janeiro, RJ: Objetiva.

Mannor, M. J., Wowak, A. J., Bartkus, V. O., \& Gomez-Mejia, L. R. (2016). Heavy lies the crown? How job anxiety affects top executive decision making in gain and loss contexts. Strategic Management Journal, 37(9), 1968-1989. doi:10.1002/smj.2425

Maslach, C., \& Jackson, S. (1981). The Maslach Burnout Inventory. Palo Alto, CA: Consulting Psychologists Press.

Maslach, C., \& Leiter, M. P. (2015). Editorial: It's time to take action on burnout. Burnout Research, 1, 1-2. doi: 10.1016/j.burn.2015.05.002

Maslach, C., Schaufeli, W. B., \& Leiter, M. P. (2001). Job burnout. Annual Review of Psychology, 52(1), 397-422. doi:10.1146/annurev.psych.52.1.397

Modesto, J. G. N. (2018). "Por que corruptos são corruptos?": Propositura e apresentação de evidências do Modelo Analítico da Corrupção. Brasília, DF: Universidade de Brasília.

Pettigrew, T. F. (2018). The emergence of Contextual Social Psychology. Personality and Social Psychology Bulletin, 44(7), 963-971. doi:10.1177/0146167218756033 
Schaufeli, W. B., Leiter, M. P., \& Maslach, C. (2009). Burnout: 35 years of research and practice. Career Development International, 14 (3), 204-220. doi:10.1108/13620430910966406

Schindler, S., \& Pfattheicher, S. (2017). The frame of the game: Loss-framing increases dishonest behavior. Journal of Experimental Social Psychology, 69, 172-177. doi:10.1016/j.jesp.2016.09.009

Schuster, M. D. S., \& Dias, V. D. V. (2018). Oldenburg Burnout Inventory-validação de uma nova forma de mensurar Burnout no Brasil. Ciência \& Saúde Coletiva, 23, 553-562. doi:10.1590/1413-81232018232.27952015.

Sokol-Hessner, P., \& Rutledge, R. B. (2019). The psychological and neural basis of loss aversion. Current Directions in Psychological Science, 28(1), 20-27. doi:10.1177/0963721418806510

Sonnentag, S., \& Fritz, C. (2007). The Recovery Experience Questionnaire: Development and validation of a measure for assessing recuperation and unwinding from work. Journal of Occupational Health Psychology, 12(3), 204-221. doi:10.1037/1076-8998.12.3.204

Squires, A., Finlayson, C., Gerchow, L., Cimiotti, J. P., Matthews, A. .... Sermeus, W. (2014). Methodological considerations when translating "burnout". Burnout Research, 1(2), 59-68. doi:10.1016/j.burn.2014.07.001

Tamayo, M. R., \& Tróccoli, B. T. (2009). Construção e validação fatorial da Escala de Caracterização do Burnout (ECB). Estudos de Psicologia, 14(3), 213-221. doi:10.1590/S1413294X2009000300005

Tversky, A., \& Kahneman, D. (1992). Advances in prospect theory: Cumulative representation of uncertainty. Journal of Risk and Uncertainty, 5(4), 297-323. doi:10.1007/BF00122574

Valle, M. A., \& Ruz, G. A. (2015). Turnover prediction in a call center: Behavioral evidence of loss aversion using random forest and naïve bayes algorithms. Applied Artificial Intelligence, 29(9), 923-942. doi:10.1080/08839514.2015.1082282

Walasek, L., \& Stewart, N. (2015). How to make loss aversion disappear and reverse: Tests of the decision by sampling origin of loss aversion. Journal of Experimental Psychology: General, 144(1), 7-11. doi:10.1037/xge0000039

Wang, M., Rieger, M. O., \& Hens, T. (2017). The impact of culture on loss aversion. Journal of Behavioral Decision Making, 30(2), 270-281. doi:10.1002/bdm.1941

\section{CONFLITOS DE INTERESSES}

Não há conflitos de interesses.

\section{SOBRE OS AUTORES}

João Gabriel Modesto é Professor DES IV (adjunto) da Universidade Estadual de Goiás e Professor Titular do Centro Universitário de Brasília, sendo que nesta instituição está vinculado ao programa de mestrado em Psicologia.

E-mail: joao.modesto@ueg.br

(1) https://orcid.org/0000-0001-8957-7233

Tatiana Rodrigues é psicóloga graduada pelo Centro Universitário de Brasília

E-mail: tatianajornalista26@gmail.com

(1) https://orcid.org/0000-0003-0430-1508

Fabiana Queiroga é professora titular do Centro Universitário de Brasília. É doutora em Psicologia Organizacional e do Trabalho pela Universidade de Brasília e psicóloga pela Universidade Federal da Paraíba. Fez pós-doutorado na Université Côte D’Azur (Nice/França) com ênfase em processos laborais associados ao desempenho sustentável.

E-mail: fabiana.queiroga@ceub.edu.br

https://orcid.org/ 0000-0002-3811-8202 